\title{
In Vitro Differentiation of Human Bone Marrow Stem Cells into Retinal Pigment Epithelium
}

\author{
Nehal Draz ${ }^{1}$, Makram F. Attalah ${ }^{1}$, Hany M. Shaheen ${ }^{1}$, Wael Abo Elkeir ${ }^{2, *}$ \\ ${ }^{1}$ Medical Microbiology and Immunology, Ain Shams University, Cairo, Egypt \\ ${ }^{2}$ Military Medical Academy, Cairo, Egypt
}

Email address:

dr.wael2008@yahoo.com

\section{To cite this article:}

Nehal Draz, Makram F. Attalah, Hany M. Shaheen, Wael Abo Elkeir. In Vitro Differentiation of Human Bone Marrow Stem Cells into Retinal Pigment Epithelium. American Journal of Bioscience and Bioengineering. Special Issue: Stem Cells for Neuro-regeneration: Where Do We Stand. Vol. 3, No. 4-1, 2015, pp. 51-55. doi: 10.11648/j.bio.s.2015030401.18

\begin{abstract}
Introduction: Dysfunction, degeneration, and loss of retinal pigment epithelial (RPE) cells are prominent features of subtypes of retinitis pigmentosa (RP) which is a cause of visual disability. In this condition, there is progressive visual loss that often leads to blindness. A variety of therapeutic approaches to delay the degenerative process are under development, the most promising of which is the stem cell regenerative therapy. Stem cells are self-renewing cells capable of transdifferentiation into a number of cell lineages. Mesenchymal stem cells (MSCs) represent a type of stem cell with remarkable proliferative, regenerative and transdifferentiation ability. Aim of the Work: The aim of this work was to evaluate the ability of bone marrow derived MSCs to differentiate into retinal pigmented epithelial cells in vitro as a step towards regeneration of degenerated retinal cells as in cases of retinitis pigmentosa. Patients and Methods: The present study was carried out on 20 bone marrow samples obtained from donors undergoing bone marrow aspirations, at EL-Maadi Military Hospital. $3 \mathrm{ml}$ bone marrow were aspirated, mesenchymal stem cells were separated and induced to RPE lineage through culture in the presence of nicotinamide NIC and factors from the TGF-b superfamily (Activin A). The BMSC- derived pigmented-like cells were evaluated for the morphology, function, and marker expression of authentic RPE using RT-PCR for RPE65 mRNA. Results: Bone marrow derived mesenchymal stem cells in all samples demonstrated the morphologic characteristics as well as molecular markers of RPE cells after 8 weeks of culture with activin A and nicotinamide. Conclusion: In the present study, compelling evidences support the possibility of differentiation of bone marrow stem cells into retinal pigmented epithelial cells (RPE) which have the morphology, function, and marker expression of authentic RPE.
\end{abstract}

Keywords: Retinal Pigment Epithelial (RPE), Retinitis Pigmentosa (RP), Nicotinamide (NIC), Activin A, Bone Marrow Derived Stem Cells (BMSC)

\section{Introduction}

The retinal pigment epithelium (RPE), a monolayer of cells between the neural retina and the choriocapillaris, is the main player in the maintenance and function of the retina and its photoreceptors. RPE is involved in the establishement of the blood retinal barrier, nutrient supply to the neural retina, absorption of stray light, visual pigment regeneration, as well as uptake and subsequent recycling of shed outer segments of photoreceptors (Strauss, 2005).

Retinitis pigmentosa (RP) is a group of inherited disorders characterized by progressive peripheral vision loss and night vision difficulties (nyctalopia) that can lead to central vision loss. RP comprises a number of retinal and retinal pigment epithelium (RPE) dystrophies. The genetic background expands to defects in more than 40 different genes. The clinical heterogeneity of RP is caused partly by the different genes implicated and partly by the phenotypic difference in gene expression (Fahim et al, 2013).

Although a variety of therapeutic approaches to delay the degenerative process were many patients eventually lose their sight. Cell therapy is introduce as a food alternative to replenish the degenerating RPE cells and potentially slow or stop disease progression (da Cruz et al., 2007).

In animal models of retinal degeneration, subretinal transplantation of RPE cells was found to rescue photoreceptors and preserve visual function (Sauve et al., 2002). Partial restoration of visual function was also reported in humans, mainly after autologous RPE layer transplantation 
(da Cruz et al., 2007).

Bone marrow stem cells represent a cell population with remarkable plastic and versatile properties because of their multipotency and their ability for multilineage differentiation both in vitro and in vivo (Bongso and Lee, 2005). Mesenchymal stem cells (MSCs) are a type of multipotent adult stem cells that are present in the bone marrow, cord blood, Wharton Jelly and adipose tissue. MSCs can be expanded into large quantities in vitro. They can be induced in vitro to differentiate into any of the mesoderm lineage, such as adipocytes, chondrocytes and osteoblasts. Moreover, transplanted BM-MSCs can generate multiple lineage cells, including brain, lung, renal, gastrointestinal tract, skin and pancreatic $\beta$-cells (Zhao et al., 2009).

The present work was designed to evaluate the ability of bone marrow derived MSCs to adopt the morphologic and functional characteristics of RPE. This is a crucial step towards its use as a regenerative tool in cases of RP.

\section{Material and Methods}

\subsection{Sampling}

The present study was carried out on 20 bone marrow donors, 15 males and 5 females between 23 and 41years old, at EL-Maadi Military Hospital after obtaining their informed consents. $3 \mathrm{ml} \mathrm{BM}$ was obtained under aseptic conditions on preservative free heparin.

\subsection{Sample Preparation}

Mononuclear fraction was collected using Ficoll-Hypaque (Sigma-Aldrich) density gradient centrifugation (Heraeus Labofuge 200- Thermo Electron Corporation-Germany- serial no. 75003630).

Mononuclear cells were plated at a density of $1 \times 10^{6}$ in tissue culture flasks T25 $\left(25 \mathrm{~cm}^{2}\right)$ using low glucose Dulbecco's modified eagle's medium (DMEM) (Lonza-Belgium) supplemented with fetal bovine serum (FBS) (10\%) (BiowestU.S.A), penicillin (100 ug/ml), streptomycin (100 ug/ml) (Lonza). Flasks were incubated for $48 \mathrm{~h}$ in $5 \% \mathrm{CO}_{2}$ humidified incubator. On day 3, non-adherent cells were discarded and medium replenished. Medium was changed every 3 days and cultured examined using the inverted microscope until $80-90 \%$ confluence, MSCs were then harvested using $0.25 \%$ trypsin-EDTA (Lonza) according to (Doyle and Bryan, 1998).

MSCs were identified according to the minimal criteria of the ISCT using plastic adherence, morphology, and immunophenotyping (Dominici et al 2006).

\subsection{Flow Cytometry}

Immunophenotyping was done using flow cytometer (Beckman Coulter Epics XL - U.S.A) for CD90 \& CD 271 and CD34.

\subsection{Retinal Cell Differentiation}

The collected MSCs were sub-cultured on tissue culture flasks containing RPE inductive medium consisting of : DMEM (Lonza) supplemented with fetal bovine serum (FBS) $(10 \%)$, penicillin /streptomycin $(100 \mathrm{ug} / \mathrm{ml})$ (Lonza), activin A $(100 \mathrm{ng} / \mathrm{ml})$ (Reagent proteins) and nicotinamide $(10 \mathrm{mM})$ (Sigma-Aldrich). Cells were incubated for an additional 8 weeks with changing medium every 4 days and doing subculture every 10 days to prevent overgrowth of cells (Maria et al., 2009).

\subsection{PCR Analysis}

Cells were collected for RT-PCR. Total RNA was extracted from differentiated bone marrow stem cells by means of RNeasy Mini Kit (QIAGEN). RT- PCR was carried out with One Step RT-PCR Kit (QIAGEN) with random primers for the expression of RPE65 gene according to the manufacturer's instructions (Maria et al., 2009, Doset al., 2014, Ma et al 1999).

\subsection{QIAGEN One Step RT-PCR Procedure}

Template RNA, primer solutions (forward primer, 5'GTTTCTGATTGTGGATCTC-3') and (reverse primer, 5'GGGATGTTAATCTCCACTTC-3' (Edgar Selzer et al., 2002), dNTP Mix, 5x QIAGEN OneStep RT-PCR Buffer, and RNase-free water were thawed, and placed on ice.

Table 1. Compenents for Molecular Identification of RPE65 mRNA.

\begin{tabular}{ll}
\hline Component & Volume/reaction \\
\hline Master mix & \\
RNase-free water (provided) & $18 \mathrm{ul}$ \\
5 X QIAGEN OneStep RT-PCR Buffer & $10 \mathrm{ul}$ \\
dNTP Mix (containing $10 \mathrm{mM}$ of each dntp) & $2 \mathrm{ul}$ \\
Forward Primer & $3 \mathrm{ul}$ \\
Reverse Primer & $3 \mathrm{ul}$ \\
QIAGEN OneStep RT-PCR Enzyme Mix & $2 \mathrm{ul}$ \\
RNase inhibitor (optional) & $2 \mathrm{ul}$ \\
Template RNA & $10 \mathrm{ul}$ \\
Total volume & $50 \mathrm{ul}$ \\
\hline
\end{tabular}

- The master mix was mixed thoroughly by pipetting up and down a few times, and $40 \mu \mathrm{g} /$ reaction was dispensed into PCR tubes.

- Template RNA (10 $\mu \mathrm{g} /$ reaction) was added to the individual PCR tubes.

Table 2. Thermal Cycler Program for RPE65 mRNA amplification

\begin{tabular}{lll}
\hline & Time & Temperature \\
\hline Reverse transcription & 30 minutes & $50^{\circ} \mathrm{C}$ \\
Initial PCR activation step: & 15 minutes & $95^{\circ} \mathrm{C}$ \\
3 step cycling: & & \\
Denaturation: & 1 minute & $94^{\circ} \mathrm{C}$ \\
Annealing: & 1 minute & $50-68^{\circ} \mathrm{C}$ \\
Extension: & 3 minute & $72^{\circ} \mathrm{C}$ \\
Number of cycles: & 45 & \\
Final extension: & 10 minutes & $72^{\circ} \mathrm{C}$ \\
\hline
\end{tabular}

\subsection{Gel Electrophoresis}

To determine whether the MSCs had differentiated into retinal pigment cells, the expression of RPE mRNA was 
examined by detection of amplified product on agarose gel (3\%) (Maria et al., 2009).

\section{Results}

\subsection{Human BM-MSCs Characterization}

1. Cell count and cell viability:

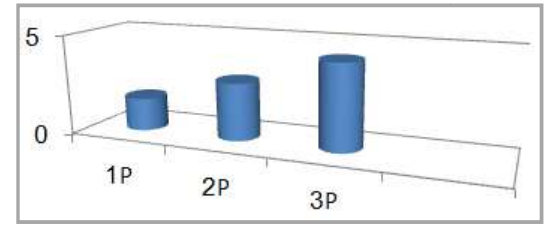

Figure 1. Histogram representing cell count in differnent passages.

Total cell count $\times 10^{6}$ cells $/ \mathrm{ml}: \quad(\mathrm{P} 1)$ of isolated mesenchymal stem cells from BM at first passage was $1 \times 10^{6}$ cells $/ \mathrm{ml},(\mathrm{P} 2)$ of cultivated mesenchymal stem cells at second passage was $2 \times 10^{6}$ cells $/ \mathrm{ml}$, and (P3) of third passage was $4 \times 10^{6}$ cells $/ \mathrm{ml}$. MSC viability was done by the reaction with Trypan Blue showing a viability of $95 \%$

\subsection{Characterization of Human BM-MSCs by Flow- cytometry}

Flow cytometry analysis showed that the cells were

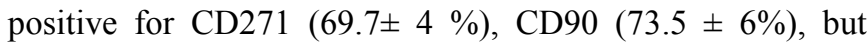
negative for CD34 $(6.6 \pm 2 \%)$ which is Hematopoietic cell marker.

\subsection{Morphology of Isolated MSCs}

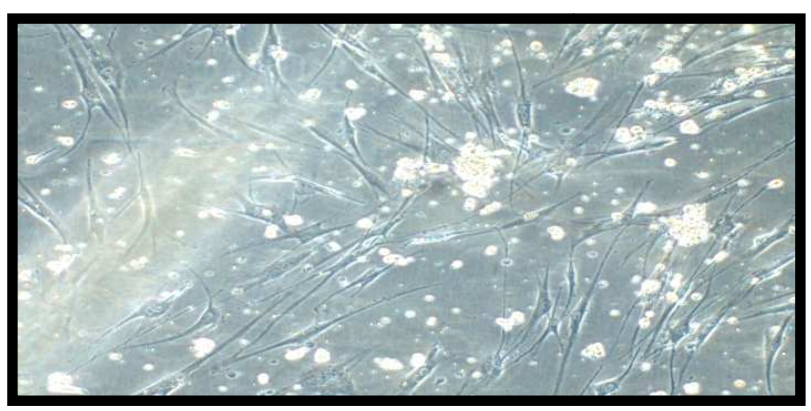

Figure 2. Inverted microscope image of MSCs at day 12, showing fibroblastoid (100x).

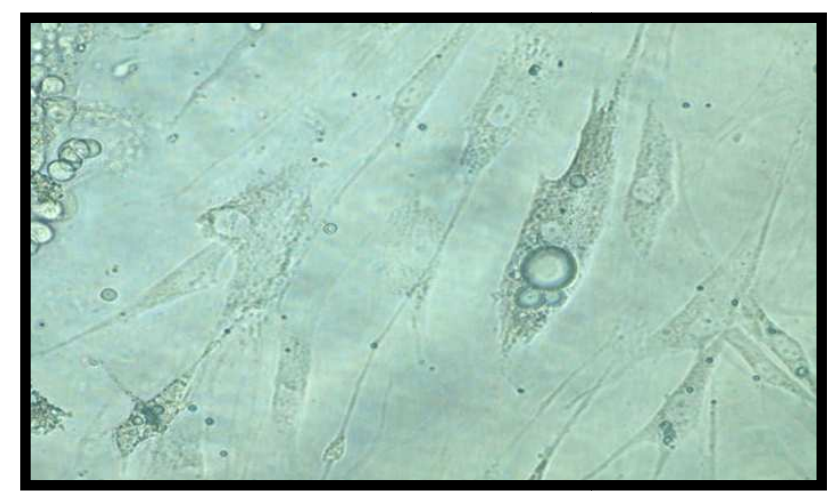

Figure 3. Inverted microscope image of MSCs at day 12, showing cellular morphology (400x).

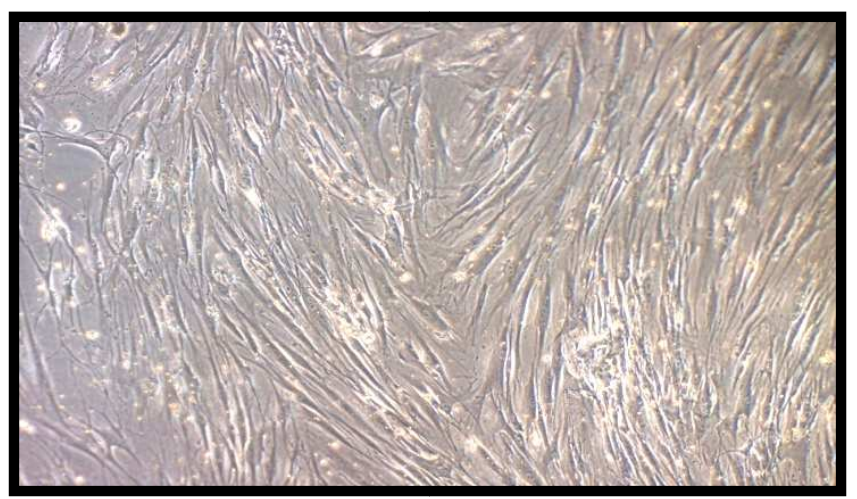

Figure 4. Inverted microscope image of MSCs showing $90 \%$ confluence $(100 X)$.

\subsection{Characterization of BM-MSCs Differentiation into Retinal Pigment Cells}

a. Morphological changes:

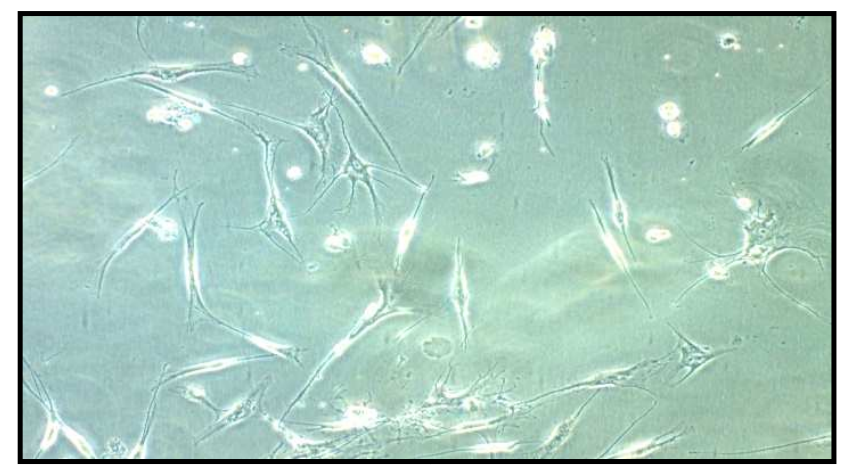

Figure 5. MSC after 4 weeks in RPE induction medium showing morphological changes in the form of multiple cellular processes and pyramidal neuron-like cells(100X).

b. Molecular identification:

All samples were cultured for 8 weeks on a special medium which was changed every 4 days then RT-PCR was done for the differentiated cells to detect RPE mRNA.

\section{Discussion}

Eyes originate from the forebrain as bilateral invaginations (optic vesicles) comprised of progenitors expressing eye field markers, which can give rise to both neural retina and RPE. Differentiation of neural retina and RPE is controlled by the activity of pax2, pax6, and MiTF genes (Baumer et al., 2003). These genes act as activators of proneural genes, become downregulated as RPE mature, remaining active in ganglion cells in mature retina (AsheryPadan and Gruss, 2001). RPE65 is an isomerohydrolase expressed in retinal pigment epithelium. It is critical for the regeneration of the visual pigment necessary for both rod and cone-mediated vision (Cai et al, 2009).

Ying and coauthors (2003) have shown that neuronal commitment is regulated by autocrine FGF (Fibroblast growth factor).The present study suggests the use of Activin A which is a member of TGF-b (tumor growth factor-b) 
superfamily as a growth factor in the culture of BMSC leads to its differentiation into retinal pigment epithelium. This result goes with the result of Anthony Kicic et al (2003) which showed that bone marrow stromal cells of rat can be differentiated into retinal pigment epithelium in the presence of Activin A.

Maria et al., (2009) have shown that NIC and Activin A can promote, under defined culture conditions, the differentiation of human embryonic stem cells (hESC) to RPE. This result goes with our present study result that NIC and Activin A can promote, under defined culture conditions, the differentiation of bone marrow stem cells (BMSC) towards an RPE fate. The BMSC-derived pigmented cells exhibit the morphology, function, and marker expression of authentic RPE.

NIC is a precursor of the coenzyme b-nicotinamide adenine dinucleotide (NAD), which functions in the production of ATP in the mitochondria. In line with our findings, NIC was demonstrated to increase cell survival (Chong et al., 2005), and has a potent neuroprotective action in vitro (Shen et al., 2004).

NIC is an inhibitor of the enzyme Poly-ADP-Ribose Polymerase (PARP), which is involved in DNA repair and cell death, including retinal cell death (Paquet-Durand et al., 2007). Therefore, it may be speculated that in our system NIC promoted neural and retinal cell survival potentially by PARP inhibition.

In the presence of NIC, the process of BMSC differentiation toward RPE was augmented and recapitulated the key steps of RPE development in vivo. Similar to embryonic eye development, in our system, NIC initially significantly promoted differentiation into RPE.

The effect of NIC on RPE differentiation was further augmented by Activin A. This is in line with the presumed role of Activin A in RPE development in vivo. In the embryo, extra ocular mesenchyme-secreted members of the TGF-b superfamily are thought to direct the differentiation of the optic vesicle into RPE (Fuhrmann et al., 2000).

Maria et al., (2009) have mentioned that the expression of transcripts of the early RPE marker MiTF-A was also augmented In the presence of NIC mainly after 4 to 8 weeks. In our study, cells were collected after 8 weeks forRT-PCR analysis and gel electrophoresis to detect MiTF-A gene.

\section{Conclusion}

Our results showed the possibility of differentiation of bone marrow stem cells into retinal pigmented epithelial cells (RPE) which have the morphology, function, and marker expression of authentic RPE in vitro.

This gives the hope to start trials on animal models to examine the possibility of bone marrow derived stem cell differentiation into RPE in vivo.

Finally, this study aimed to prove that stem cells, with properties of self-renewal, will offer an ideal donor source for RPE transplantation in some cases of untreatable retinal diseases as retinitis pigmentosa in the future.

\section{References}

[1] Bongso A., and Eng Hin Lee (2005): stem cells from bench beside. ISBN: 978-981-4481-16-8 (ebook).Development 127, $4599-4609$.

[2] Cai X, Conley S, Naash M (2009): RPE65: Role in the visual cycle, human retinal disease, and gene therapy. Ophthalmic Genet. 2009 Jun; 30(2): 57.

[3] Chong Z.Z, Lin S.H, Li F and Maiese K (2005): The sirtuin inhibitor nicotinamide enhances neuronal cell survival during acute anoxic injury through AKT, BAD, PARP, and mitochondrial associated "anti-apoptotic" path ways. Curr. Neurovasc. Res. 2, 271-285.

[4] da Cruz, L., Chen, F.K., Ahmado, A., Greenwood, J., and Coffey, P. (2007). RPE transplantation and its role in retinal disease. Prog. Retin. Eye Res. 26, 598-635.

[5] Dominici $\mathrm{M}^{1}$, Le Blanc K, Mueller I, Slaper-Cortenbach I, Marini F, Krause D, Deans R, Keating A, Prockop Dj, Horwitz E.(2006): Minimal criteria for defining multipotent mesenchymal stromal cells. The International Society for Cellular Therapy position statement. Cytotherapy. 2006;8(4):315-7.

[6] Doyle A \& J.Bryan Griffiths (1998): Cell and Tissue Culture: Laboratory Procedures in Biotechnology. ISBN: 978-0-47198255-5.

[7] Fahim A, Daiger S, Weleber R (2000): Retinitis Pigmentosa Overview. GeneReviews Advanced Search

[8] Fuhrmann S, Levine E.M and Reh T.A (2000): Extraocular mesenchyme patterns the optic vesicle during early eye development in the embryonic chick. Development 127, 4599-4609.

[9] Fuhrmann, S., Levine, E.M., and Reh, T.A. (2000). Extraocular mesenchyme

[10] Ma J, Zhang D, Laser M, Brownlee N, Re G, Hazen-Martin D, Redmond T, Crouch R (1999): Identification of RPE65 in transformed kidney cells. FEBS Letters 452(3):199-204.

[11] Maria Idelson, Ruslana Alper, Alexey Obolensky, Etti BenShushan, Itzhak Hemo, Nurit Yachimovich-Cohen, et al (2009): Directed Differentiation of Human Embryonic Stem Cells into Functional Retinal Pigment Epithelium Cells. Cell Stem Cell 5, 396-408.

[12] Paquet-Durand F, Silva J, Talukdar T, Johnson L.E, Azadi S, van Veen T, Ueffing M, Hauck S.M and Ekstrom P.A (2007): Excessive activation of poly (ADP-ribose) polymerase contributes to inherited photoreceptor degeneration in the retinal degeneration 1 mouse. J. Neurosci. 27, 10311-10319. patterns the optic vesicle during early eye development in the embryonic retinal pigment epithelium cell transplantation. Neuroscience 114, 389-401.

[13] Sauvé (2002): Neuroscience, 114(2):389-401.

[14] Sauve, Y., Girman, S.V., Wang, S., Keegan, D.J., and Lund, R.D. (2002). Preservation of visual responsiveness in the superior colliculus of RCS rats after

[15] Shen C.C, Huang H.M, Ou H.C, Chen H.L, Chen W.C and Jeng K.C (2004): Protective effect of nicotinamide on neuronal cells under oxygen and glucose deprivation and hypoxia/reoxygenation. J. Biomed. Sci. 11, 472-481. 
[16] Strauss O (2005): The retinal pigment epithelium in visual function. Physiol.Rev. 85, 845-881.
[17] Zhang Z, Zhang Y, Xiao H, Liang X, Sun D, Peng S.(2012): A gene expression profile of the developing human retinal pigment epithelium Mol Vis. 2012;18:2961-75. 